\title{
Locally Advanced Colorectal Cancer: True Peritoneal Tumor Penetration is Associated with Peritoneal Metastases
}

\author{
Charlotte E. L. Klaver, MD ${ }^{1}$, Nadine C. M. van Huijgevoort, $\mathbf{M D}^{1}$, Anthony de Buck van Overstraeten, $\mathbf{M D}^{3}$, \\ Albert M. Wolthuis, MD, $\mathbf{P h D}^{3}$, Pieter J. Tanis, $\mathrm{MD}, \mathbf{P h D}^{1}$, Jarmila D. W. van der Bilt, $\mathbf{M D}, \mathbf{P h D}^{1,3,4}$, \\ Xavier Sagaert, $\mathrm{MD}, \mathbf{P h D}^{2}$, and André D'Hoore, $\mathbf{M D}, \mathbf{P h D}^{3}$ \\ ${ }^{1}$ Department of Surgery, Academic Medical Centre, University of Amsterdam, Amsterdam, The Netherlands; ${ }^{2}$ Department \\ of Pathology, UZ Leuven, Louvain, Belgium; ${ }^{3}$ Department of Abdominal Surgery, UZ Leuven, KU Leuven, Louvain, \\ Belgium; ${ }^{4}$ Department of Surgery, Flevoziekenhuis, Almere, The Netherlands
}

\begin{abstract}
Background. Findings show T4 colorectal cancer (CRC) to be a risk factor for the development of peritoneal metastases (PM). Heterogeneity regarding peritoneal involvement of T4 tumors might explain the wide range of reported PM incidences (8-50\%). Hyperplastic and mesothelial inflammatory reactions complicate evaluation of the exact primary tumor involvement of the peritoneal layer. This retrospective cohort study aimed to assess the association between either inflammatory peritoneal reaction or peritoneal involvement of the primary tumor and the risk of PM.

Methods. Since 2010, pathologists at UZ Leuven have systematically categorized peritoneal involvement in peritoneal reaction with tumor less than $1 \mathrm{~mm}$ from the peritoneal surface or true peritoneal penetration. All patients undergoing resection of CRC between January 2010 and July 2013 who fulfilled either of these pathologic criteria were included in this study.

Results. The study enrolled 159 CRC patients. Peritoneal reaction with tumor less than $1 \mathrm{~mm}$ from the peritoneal surface was present in 43 patients and true peritoneal penetration in 116 patients. Overall, 29 patients (18\%) had
\end{abstract}

Electronic supplementary material The online version of this article (doi:10.1245/s10434-017-6037-6) contains supplementary material, which is available to authorized users.

(C) The Author(s) 2017. This article is an open access publication

First Received: 29 March 2017;

Published Online: 26 October 2017

C. E. L. Klaver, MD

e-mail: c.e.klaver@amc.nl synchronous PM, and 30 patients (23\%) had metachronous $\mathrm{PM}$. In the multivariable analysis, true peritoneal penetration, in contrast to peritoneal reaction with tumor less than $1 \mathrm{~mm}$ from the peritoneum, was associated with greater risk of PM (odds ratio [OR], 2.518; range, 1.038-6.111; $p=0.041)$ and lymph node involvement (N1: OR, 1.572; range, $0.651-3.797$ vs $\mathrm{N} 2$ : $\mathrm{OR}, \quad 4.046$; range, $1.549-10.569 ; p=0.014)$.

Conclusion. Histologically confirmed true peritoneal penetration by $\mathrm{CRC}$, rather than inflammatory peritoneal reaction constitutes a high risk for PM. With evolving treatment strategies that aim to treat PM in an earlier phase, identification of high-risk patients becomes highly important clinically.

Locally advanced (stage T4) colorectal cancer (CRC) is subdivided into T4a (penetration of the visceral peritoneum) and T4b (adjacent organ invasion) (Table S1, tumor-nodemetastasis [TNM]7) and constitutes a risk factor for the development of peritoneal metastases $(\mathrm{PM}) .{ }^{1-5}$ It is hypothesized that PM development is a consequence of malignant cells detached from the primary T4 tumor entering the peritoneal cavity. Subsequently, these free cells attach to the peritoneal surface and progress into PM. ${ }^{6,7}$

The reported risks for the development of PM in T4 CRC vary widely, from 8 to $50 \%$. This variation can be partly explained by the heterogeneity of $\mathrm{T} 4$ tumors with respect to local peritoneal involvement. Hyperplastic and mesothelial inflammatory reactions of the peritoneum often complicate evaluation of the exact tumor involvement of the very thin peritoneal layer. As a consequence, it cannot be clearly determined whether a certain group of CRC tumors should be classified as $\mathrm{T} 3$ or $\mathrm{T} 4$. 
In 2002, a narrow definition of T4 was determined for TNM6, ${ }^{8}$ encompassing tumors that perforate the visceral peritoneum. In 2006, Compton ${ }^{9}$ proposed a wider definition of $\mathrm{T} 4$ that also included hyperplastic and inflammatory reactions of the peritoneum. Since then, different interpretations of pT4 among pathologists have been used.

Conceivably, true tumor penetration of the peritoneum would result in the highest risk for PM. Shepherd et al. ${ }^{10,11}$ earlier showed the prognostic importance of subcategorizing tumors based on the extent of local peritoneal involvement (LPI; Table S2), distinguishing hyperplastic reactions, mesothelial inflammation, and true penetration of the peritoneum. However, few patient data exist on the risk for PM in these subcategories, probably due to restricted therapeutic consequences.

Currently, attention for T4 stage colon cancer is growing, with studies aiming to prevent the development of PM by prophylactic hyperthermic intraperitoneal chemotherapy (HIPEC) $)^{12}$ or to detect PM at an early but still curable stage by second-look surgery. This study aimed to investigate the association between true peritoneal penetration of the primary tumor, in contrast to peritoneal reaction with tumor very close to the peritoneum (within $1 \mathrm{~mm}$ ), and the development of PM in CRC.

\section{METHODS}

\section{Patients}

Patients were selected from a CRC database of the University Hospital Leuven (UZ Leuven). Since 2010, pathologists of UZ Leuven choose to define pT4 as true peritoneal tumor penetration or peritoneal hyperplastic of inflammatory reaction with tumor less than $1 \mathrm{~mm}$ from the peritoneum, in line with the considerations of Compton. ${ }^{9}$ They have systematically subclassified locally advanced tumors as true peritoneal tumor penetration, as peritoneal reaction with tumor less than $1 \mathrm{~mm}$ from the peritoneum, or as tumor without peritoneal involvement (i.e., tumors invading adjacent retroperitoneal organs or structures).

The current study enrolled all patients with an intraperitoneally located primary CRC (above the peritoneal reflection) undergoing resection between January 2010 and July 2013 with either peritoneal tumor penetration or peritoneal reaction. If the peritoneal involvement was not systematically scored in the pathology report, the patient was excluded from the study.

\section{Pathology}

Per colorectal cancer specimen, one formalin-fixed, paraffin-embedded (FFPE) block per centimeter of tumor was collected (at random locations). From all areas with macroscopic suspicion of deepest ingrowth, a separate block was collected. Between two and five slides were retrieved per block to determine deepest penetration. In case a tumor within $1 \mathrm{~mm}$ of the peritoneal surface was detected, at least three deeper slides were retrieved from the relevant block to detect potential true tumor penetration. In case of hesitation, more material was collected. True tumor penetration was defined as tumor cells at the peritoneal surface or free tumor cells on the peritoneum with underlying ulceration of the peritoneum.

\section{Variables}

Synchronous PM was defined as the presence of PM at the time of CRC diagnosis. Metachronous PM was defined as PM diagnosed during routine follow-up assessment by any methods or combination of methods such as imaging, re-laparotomy, or both.

Perforation refers to tumor or bowel perforation or suspected perforation based on clinical or intraoperative findings. Radicality of resection is subcategorized into three groups: R0 (radical resection with $>1$-mm tumor-free margin), R1 (microscopically nonradical resection involving $\leq 1$-mm margin), and R2 (macroscopically nonradical resection).

\section{Statistical Analysis}

Baseline characteristics are expressed as counts and percentages. Differences in baseline characteristics between groups were analyzed using a Chi square test or Fisher's exact test. For normally distributed continuous variables, mean and standard deviation are given, and for non-normally distributed continuous variables, median and interquartile range (IQR) are reported.

Using logistic regression, independent factors associated with PM were identified. For the purpose of this analysis, a combined variable of synchronous and metachronous PM was used. Patients lost to follow-up evaluation without signs of PM within 3 years were categorized as "unknown." Variables that were significant independent predictive factors in the univariable analysis $(p \leq 0.10)$ were included in the multivariable regression analysis. In the multivariable analysis, multicollinearity was assessed.

Association with metachronous PM was evaluated using Kaplan-Meier curves and Cox regression. Kaplan-Meier curves were truncated when numbers at risk became less than one-third of the starting group. For this analysis, patients with synchronous PM were excluded. Again, factors significantly associated with metachronous PM $(p \leq 0.10)$ in the univariable analysis were included in the multivariable analysis. In the multivariable analysis, a 
$p$ value of 0.05 or lower was considered statistically significant. Because patients with radical resected primary T4 tumors that had no synchronous peritoneal or distant metastases are considered potentially eligible for studies on prophylactic therapy, the Cox regression analysis was repeated for this subgroup. Statistical analyses were performed using PASW Statistics, version 22 (SPSS Inc., Chicago, IL, USA).

\section{RESULTS}

\section{Patients}

Between January 2010 and July 2013, 973 patients underwent surgery for primary CRC at UZ Leuven. Locally advanced tumor was diagnosed for 183 of these patients, fulfilling one of the histologic criteria used by the pathologists of UZ Leuven since 2010. The study excluded 17 patients because the pathologic peritoneal involvement was not systematically scored. Another seven patients were excluded because the primary tumor did not involve the peritoneum. Of the 159 patients included in this analysis, $43 \mathrm{had}$ "peritoneal reaction with tumor less than $1 \mathrm{~mm}$ from the peritoneal surface" and 116 had "true peritoneal tumor penetration" (Fig. S1).

The mean age of the patients was $69 \pm 13$ years. For the majority of the patients, the diagnosed tumor was located in the recto sigmoid (44\%) or the ascending colon $(28 \%)$. All the patients had a diagnosis of adenocarcinoma, with a mucinous component in $26 \%$ of the patients. Of all the patients, $75 \%$ had surgery in the elective setting, and $42 \%$ underwent an initially laparoscopic resection. At time of diagnosis, $62 \%$ of the patients had lymph node metastases, and 36\% had distant metastases. The median followup period was 35 months (IQR, 15-48 months), and the median overall survival time was 19 months (IQR, 11-31 months). Baseline characteristics were comparable between the two histologic subgroups (Table 1).

\section{Incidences of $P M$}

Of the 159 patients, $29(18 \%)$ had PM at the time the primary tumor (synchronous PM) was diagnosed (Fig. 1). All these patients underwent cytoreductive surgery (CRS), 10 of whom had surgery combined with HIPEC. Altogether, 18 patients had solitary PM, and 11 of the 29 patients also had other distant metastases diagnosed (10 with liver metastasis and 1 with lung and distant nodal metastases) at the time the primary tumor was diagnosed. Of the 130 patients without synchronous PM, 30 (23\%) experienced metachronous PM during the follow-up period. The median time to diagnosis of metachronous PM was 17 months (IQR, 8-27 months), and $90 \%$ of metachronous PM was detected within 3 years. Of the patients who had peritoneal reaction with tumor less than $1 \mathrm{~mm}$ from the peritoneal surface, $4(9.3 \%)$ presented with synchronous PM, and 5 (13\%) experienced metachronous PM. Of the patients with true peritoneal tumor penetration, 25 (22\%) presented with synchronous PM, and $25(28 \%)$ experienced metachronous PM.

\section{Risk Factors for Synchronous and/or Metachronous $P M$}

The results of logistic regression are displayed in Table 2. For this analysis, the PM status of 16 patients was categorized as "unknown" because they were lost to follow-up evaluation within 3 years with no signs of PM. Of the remaining 143 patients, $59(41 \%)$ had PM diagnosed at some point in the course of their disease (synchronous/ metachronous), and 84 (59\%) did not experience PM during their follow-up period of at least 3 years or until death.

In the univariable regression analysis, female gender, true peritoneal penetration, lymph node involvement, and synchronous distant metastases other than PM were significantly associated with PM. These variables were included in a multivariable analysis, with true peritoneal penetration (odds ratio $[\mathrm{OR}], 2.518 ; 95 \%$ confidence interval $[\mathrm{CI}], 1.038-6.111 ; p=0.041)$ and lymph node involvement (N1: OR, 1.572; 95\% CI, 0.651-3.797 vs N2: OR, 4.046; 95\% CI, 1.549-10.569; $p=0.014$ ) remaining significantly associated with PM.

\section{Risk factors for Metachronous PM}

Table S3 presents the results of the Cox regression analysis for development of metachronous PM in the subgroup of 130 patients without synchronous PM. In the univariable analysis, gender, peritoneal involvement, lymph node involvement, and synchronous distant metastases other than PM were significant predictive factors. None of these variables remained significantly associated with metachronous PM in the multivariable analysis.

In Fig. 2, the Kaplan-Meier curve shows the proportion of PM over time for the two histologic subgroups regarding peritoneal involvement. For true peritoneal penetration, the 5 -year PM proportion is $33 \%$, as opposed to $21 \%$ for peritoneal reaction with tumor less than $1 \mathrm{~mm}$ from the peritoneum $(p=0.057)$. Also Kaplan-Meier curves showing the development of PM for subgroups based on lymph node status are displayed (Fig. 3a), showing 5-year PM proportions of $23 \%$ for N0-stage, $27 \%$ for N1-stage, and $48 \%$ for N2-stage disease $(p=0.015)$. Figure $3 b$ shows the proportion of PM over time for the subgroups 
TABLE 1 Patient, tumor, and surgical characteristics

\begin{tabular}{|c|c|c|c|c|c|}
\hline & \multicolumn{2}{|c|}{$<1 \mathrm{~mm}(n=43)$} & \multicolumn{2}{|c|}{ Penetration $(n=116)$} & \multirow[t]{2}{*}{$p$ value } \\
\hline & Counts & $\%$ & Counts & $\%$ & \\
\hline \multicolumn{6}{|l|}{ Gender } \\
\hline Male & 21 & 49 & 53 & 46 & \multirow[t]{2}{*}{0.724} \\
\hline Female & 22 & 51 & 63 & 54 & \\
\hline \multicolumn{6}{|l|}{ Age (years) } \\
\hline$<60$ & 7 & 16 & 25 & 22 & \multirow[t]{4}{*}{0.281} \\
\hline $60-70$ & 14 & 33 & 33 & 28 & \\
\hline $70-80$ & 8 & 19 & 34 & 29 & \\
\hline$>80$ & 14 & 33 & 24 & 21 & \\
\hline \multicolumn{6}{|l|}{ ASA } \\
\hline $1-2$ & 26 & 68 & 77 & 73 & \multirow[t]{2}{*}{0.563} \\
\hline $3-4$ & 12 & 32 & 28 & 27 & \\
\hline \multicolumn{6}{|l|}{ Location } \\
\hline Colon & 40 & 93 & 106 & 91 & \\
\hline Rectum & 3 & 7 & 10 & 9 & \\
\hline \multicolumn{6}{|l|}{ Right versus left location } \\
\hline Right colon & 15 & 41 & 34 & 34 & \multirow[t]{3}{*}{0.138} \\
\hline Transverse colon & 6 & 16 & 7 & 7 & \\
\hline Left colon/rectum & 16 & 43 & 59 & 59 & \\
\hline Emergency setting & 7 & 17 & 31 & 27 & 0.183 \\
\hline \multicolumn{6}{|l|}{ Approach } \\
\hline Laparoscopic & 15 & 35 & 31 & 27 & \multirow[t]{3}{*}{0.395} \\
\hline Converted & 7 & 16 & 14 & 12 & \\
\hline Open & 21 & 49 & 70 & 61 & \\
\hline HIPEC & 1 & 2.3 & 10 & 8.6 & 0.165 \\
\hline Perforation & 2 & 4.7 & 15 & 13 & 0.133 \\
\hline \multicolumn{6}{|l|}{ Lymph node involvement } \\
\hline N0 & 21 & 49 & 39 & 34 & \multirow[t]{3}{*}{0.136} \\
\hline N1 & 15 & 35 & 42 & 37 & \\
\hline $\mathrm{N} 2$ & 7 & 16 & 34 & 30 & \\
\hline Synchronous distant metastases other than PM & 6 & 14 & 31 & 27 & 0.091 \\
\hline \multicolumn{6}{|l|}{ Neoadjuvant therapy } \\
\hline None & 37 & 86 & 102 & 90 & \multirow[t]{3}{*}{0.745} \\
\hline Chemotherapy & 4 & 9.3 & 7 & 7.0 & \\
\hline (Chemo)radiotherapy & 2 & 4.7 & 4 & 3.5 & \\
\hline Adjuvant chemotherapy & 22 & 54 & 77 & 70 & 0.060 \\
\hline Mucinous (component) & 9 & 21 & 32 & 28 & 0.394 \\
\hline \multicolumn{6}{|l|}{ Grade } \\
\hline Poorly & 7 & 17 & 27 & 25 & \multirow[t]{3}{*}{0.442} \\
\hline Moderately & 31 & 74 & 68 & 63 & \\
\hline Well & 4 & 9.5 & 13 & 12 & \\
\hline \multicolumn{6}{|l|}{ Radicality } \\
\hline R0 & 43 & 100 & 107 & 93 & \multirow[t]{3}{*}{0.076} \\
\hline R1 & 0 & 0 & 8 & 7.0 & \\
\hline $\mathrm{R} 2$ & 0 & 0 & 0 & 0 & \\
\hline
\end{tabular}


TABLE 1 continued

\begin{tabular}{|c|c|c|c|c|c|}
\hline & \multicolumn{2}{|c|}{$<1 \mathrm{~mm}(n=43)$} & \multicolumn{2}{|c|}{ Penetration $(n=116)$} & \multirow[t]{2}{*}{$p$ value } \\
\hline & Counts & $\%$ & Counts & $\%$ & \\
\hline \multicolumn{6}{|c|}{ Peritoneal metastases (PM) } \\
\hline Synchronous & 4 & 9.3 & 25 & 22 & 0.076 \\
\hline Metachronous & 5 & 13 & 25 & 28 & 0.069 \\
\hline
\end{tabular}

ASA American Society of Anesthesiologists; HIPEC hyperthermic intraperitoneal chemotherapy, $P M$ peritoneal metastases of colorectal origin, $R 0$ radical resection with $>1$-mm tumor-free margin, $R 1$ microscopically non-radical resection ( $\leq 1 \mathrm{~mm}$ margin), $R 2$ macroscopically non-radical resection

Baseline characteristics displayed for the subgroups: (1) peritoneal reaction with tumor less than $1 \mathrm{~mm}$ from the peritoneal surface (“< $<\mathrm{mm”)}$ and (2) true peritoneal tumor penetration ("penetration")

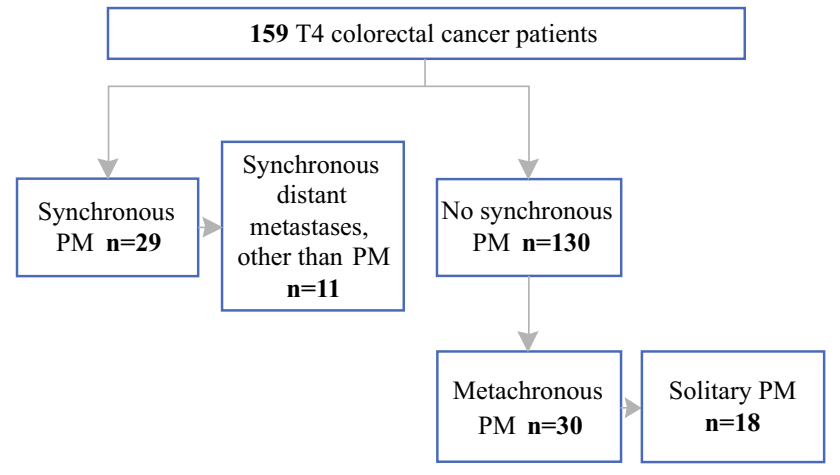

FIG. 1 Incidences of peritoneal metastases (PM) of colorectal origin

based on both peritoneal involvement and lymph node status.

Of the 159 CRC patients in this study, 104 were staged with T4NxM0 disease. For 99 of these patients, the tumor was resected radically (R0), and this subgroup could have been eligible for prophylactic therapy (i.e., adjuvant HIPEC) or second-look surgery. Metachronous PM developed for 22 (22\%) of the 99 patients in this subgroup. In the univariable analysis, female gender, peritoneal penetration, and lymph node involvement were significantly associated with the development of PM. Peritoneal penetration did not remain significantly associated with PM in the multivariable analysis (hazard ratio [HR], 2.094; 95\% CI, 0.697-6.287; $p=0.188$ ), whereas N2 stage disease did remain significantly associated with PM (N2: HR, 3.134; 95\% CI, 1.120-8.764; $p=0.030$ ).

\section{DISCUSSION}

This study showed an overall risk of $37 \%$ for the development of PM at some point in the course of disease for CRC patients with tumors nearby or penetrating the peritoneum. True peritoneal penetration, in contrast to peritoneal reaction with tumor less than $1 \mathrm{~mm}$ from the peritoneum (systematically distinguished by dedicated pathologists), was significantly associated with the overall development of PM. Also, lymph node involvement was an independent risk factor for the development of PM in this study.

For metachronous PM, peritoneal penetration was shown to be a significant risk factor only in the univariable analysis. This might have been due to restricted statistical power, although this was one of the largest cohorts reported on this subject. The 5-year risk of metachronous PM was $33 \%$ for the patients with tumors that had true peritoneal penetration and $21 \%$ for the patients with tumors that had peritoneal reaction with tumor less than $1 \mathrm{~mm}$ from the peritoneum. Both proportions are substantially higher than the 5 -year risk of $10 \%$ for patients with $\mathrm{T} 3$ colon tumors reported by Hompes et al. ${ }^{13}$ To explore very high-risk subgroups, lymph node status and peritoneal involvement were combined, showing a 55\% 5-year risk of metachronous PM for the patients with true peritoneal tumor penetration and N2-stage disease.

In the current $\mathrm{T} 4 \mathrm{a} / \mathrm{b}$ staging systems, careful evaluation of the exact peritoneal involvement is not taken into account. In cases of $\mathrm{T} 4 \mathrm{~b}$, the peritoneum (serosa) may not be involved (e.g., in retroperitoneally located tumors in the ascending or descending colon or in rectal tumors distal to the peritoneal fold). Also, the definitions have varied over time. According to TNM5, T4a is defined as "extension into nearby structures" and T4b as "perforation of the bowel." With respect to perforation, no distinction between perforation of the tumor and bowel perforation proximal to an obstructive tumor is made. In the latter subgroup, peritoneal involvement of the tumor is mostly absent.

In the literature, the association between peritoneal penetration and the development of PM often is assessed using $\mathrm{T} 4 \mathrm{a} / \mathrm{b}$ subcategorization. A subgroup of T4a colorectal carcinomas (TNM7) has been shown to have a higher metachronous PM rate (50\%, 7 of 14 patients) than a T4b subgroup (20\%, 1 of 5 patients). ${ }^{13}$ However, another study with a larger number of T4 patients $(n=200)$ 
TABLE 2 Factors associated with peritoneal metastases (PM) of colorectal origin at any point in time (synchronous/metachronous)

\begin{tabular}{|c|c|c|c|c|}
\hline & \multicolumn{2}{|l|}{ Univariable } & \multicolumn{2}{|l|}{ Multivariable } \\
\hline & OR $(95 \% \mathrm{CI})$ & $p$ value & OR $(95 \% \mathrm{CI})$ & $p$ value \\
\hline Male gender (ref: female) & $0.491(0.249-0.970)$ & $0.041^{*}$ & $0.531(0.256-1.104)$ & 0.090 \\
\hline Age (years) $($ ref $<60)$ & & 0.226 & & \\
\hline $60-70$ & $0.864(0.342-2.180)$ & & & \\
\hline $70-80$ & $0.696(0.271-1.784)$ & & & \\
\hline$>80$ & $0.348(0.120-1.006)$ & & & \\
\hline Peritoneal penetration (ref: $<1 \mathrm{~mm}$ ) & $2.929(1.264-6.787)$ & $0.012^{*}$ & $2.518(1.038-6.111)$ & $0.041^{*}$ \\
\hline Grade (ref: well differentiated) & & 0.664 & & \\
\hline Moderately & $0.789(0.211-2.951)$ & & & \\
\hline Un/poorly Diff & $0.618(0.184-2.072)$ & & & \\
\hline \multicolumn{5}{|l|}{ Mucinous component } \\
\hline Partially/yes & $1.754(0.835-3.688)$ & 0.138 & & \\
\hline $\mathrm{N}$ stage (ref: N0) & & $0.002 *$ & & \\
\hline$N=1$ & $1.827(0.787-4.240)$ & & $1.572(0.651-3.797)$ & 0.315 \\
\hline$N=2$ & $5.220(2.105-12.943)$ & & $4.046(1.549-10.569)$ & $0.004 *$ \\
\hline Synchronous distant metastases, other than PM & $2.019(0.934-4.365)$ & $0.074^{*}$ & $1.431(0.606-3.378)$ & 0.413 \\
\hline Rectum (ref: colon) & $1.463(0.404-5.299)$ & 0.562 & & \\
\hline Left-side location of tumor (ref: right) & $0.701(0.352-1.397)$ & 0.313 & & \\
\hline Emergency surgery (ref: elective) & $1.032(0.469-2.271)$ & 0.938 & & \\
\hline R1 resection (ref: R0) & $1.473(0.287-7.566)$ & 0.643 & & \\
\hline
\end{tabular}

Peritoneal penetration: true peritoneal tumor penetration

*Statistically significant

$O R$ odds ratio; $C I$ confidence interval, $<1 \mathrm{~mm}$, peritoneal reaction with tumor less than $1 \mathrm{~mm}$ from the peritoneal surface; $R 1$ microscopically non-radical resection ( $\leq 1$-mm margin); $R O$ radical resection with $>1$-mm tumor-free margin

showed no difference in synchronous (23 vs. $24 \%$ ) or metachronous ( 24 vs. $17 \%$ ) PM between patients with T4a and T4b tumors, ${ }^{14}$ nor did other studies. ${ }^{15}$ To improve risk stratification, a more careful description of the extent of peritoneal involvement should be incorporated into our T4a/T4b staging system.

Panarelli et al. ${ }^{16}$ and Snaebjornsson et al. ${ }^{17}$ recently elucidated the controversy concerning the $\mathrm{T} 4$ definition from a pathologic perspective. Snaebjornsson et al. ${ }^{17}$ reassessed pathologic T-stage and the LPI score in a nationwide colon cancer cohort $(n=889)$ and confirmed the high prognostic impact of the pT4 stage, even over lymph node involvement. He brought the definition of T4 into discussion with regard to the LPI score. It remains controversial what LPI score should be regarded as T4. These authors showed that T4b tumors (TNM7) with true peritoneal penetration (LPI4) have the worst survival rate and should be regarded as a separate category. Also, they showed that the addition of LPI3 tumors to the T4a category clearly improves the survival rate for T4a patients. They did not find a survival difference between "true" T4a (T4a + LPI4, peritoneal penetration) and "true" T4b (T4b + LPI1-3, organ involvement without peritoneal penetration) tumors. However, these authors did not separately assess development of PM, for which the LPI score might have been of particular value.

Lymph node involvement also was identified as an independent risk factor for PM in this study. This finding was confirmed in the literature. ${ }^{2,3,5,14}$ However, considering metachronous PM, the value of lymph node involvement as an independent risk factor remains controversial. $^{2-4,14,18}$ Also in this study, lymph node involvement was significantly associated with metachronous PM only in the univariable analysis, and lost its significance in the multivariable analysis due to the smaller sample in this sub-analysis.

Kaplan-Meier curves (Fig. 3a) show that proportions of $\mathrm{PM}$ increase with $\mathrm{N}$ stage, reaching the highest proportion (49\%) in N2-stage patients (HR, 3.206; 95\% CI, $1.348-7.627 ; p=0.008)$. These findings suggest an association between lymph node positivity and peritoneal metastases. It could be hypothesized that dissection of lymphatic vessels during colectomy could lead to an intraperitoneal tumor spill. Another explanation could be the existence of a CRC subtype with a high metastatic potential, causing both lymph node and peritoneal metastases.

Both adjuvant HIPEC and CRS/HIPEC are applied only when other distant metastases have not developed, or in 
FIG. 2 Metachronous peritoneal metastases (PM) of colorectal origin: the effect of peritoneal involvement.

Development of metachronous PM in patients who had T4 colorectal cancer with (1) true peritoneal tumor penetration ("penetration") and (2) peritoneal reaction with tumor less than $1 \mathrm{~mm}$ from the peritoneal surface (“<1 mm”) ( $p=0.057$, log-rank).

Truncation at 43 months, when the patients at risk became less than one-third of the starting group

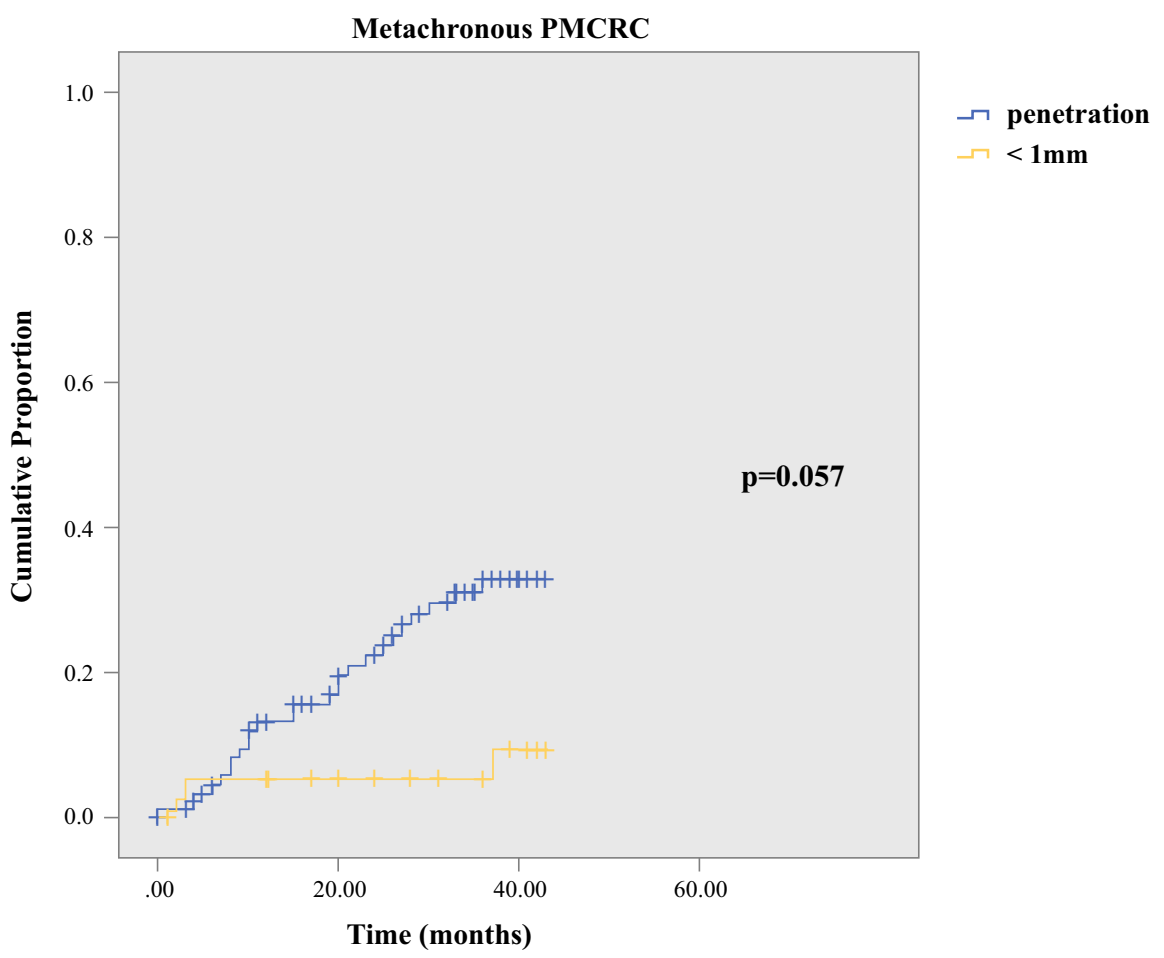

$\begin{array}{rr}\text { 0 } & 12 \\ 91 & 70 \\ 39 & 35\end{array}$

$\begin{array}{ll}12 & 24 \\ 70 & 57 \\ 35 & 28\end{array}$

$\begin{array}{ll}\mathbf{2 4} & \mathbf{3 6} \\ 57 & 39 \\ 28 & 25\end{array}$

$\begin{array}{rr}\mathbf{4 8} & \mathbf{6 0} \\ 20 & 8 \\ 11 & 0\end{array}$

case of limited resectable liver metastases. Other distant metastases also were synchronously diagnosed for $38 \%$ ( $n=11$ ) of the patients with synchronous PM in this study (Fig. 1), compromising their eligibility for CRS/HIPEC. For $40 \%(n=12)$ of the patients presenting with metachronous PM, the peritoneum was the only affected site. This subgroup in particular might benefit from the new treatment strategies.

The patient database of this study was prospectively kept with systematic scoring of the peritoneal involvement by the pathologists. However, other variables and longterm disease outcomes were retrospectively collected. The restricted sensitivity of imaging methods for the small peritoneal nodules, ${ }^{19,20}$ together with the retrospective character of this study, probably resulted in an underestimation of PM incidences. Also, because the UZ Leuven is a tertiary referral center, the study population reflected a selected (high-risk) patient group, and some patients were lost to follow-up evaluation because they were followed up in the referring hospital. Furthermore, although selection of blocks and slides from the colorectal cancer specimen during the pathologist's workup has been standardized, collection of "sufficient" material remains arbitrary to some extent. True peritoneal tumor penetration might have been missed in some of the cases that had peritoneal reaction with tumor less than $1 \mathrm{~mm}$ from the tumor.

In conclusion, histologic confirmation of true peritoneal tumor penetration by CRC constitutes a high-risk subset of tumors regarding the development of PM. Based on this finding, incorporating evaluation of the exact peritoneal tumor involvement in the tumor-node-metastasis (TNM) classification should be considered. For the patients with true peritoneal tumor penetration and N2 stage, a 5-year risk of 55\% for metachronous PM was found in this study. This group in particular might be eligible for new treatment strategies that aim to treat PM in an earlier or even preventive setting. However, the results of trials investigating these strategies should be awaited. 
FIG. 3 Metachronous peritoneal metastases (PM) of colorectal origin. a The effect of $\mathrm{N}$ stage. Development of metachronous PM in patients with $\mathrm{T} 4$ colorectal cancer in stages N0, N1, and N2 $(p=0.015$, log-rank).

Truncation at 43 months, when the patients at risk became less than one-third of the starting group. b Combined effect of $\mathrm{N}$ stage and peritoneal involvement. Development of metachronous PM in patients who had T4 colorectal cancer with (1) true peritoneal tumor penetration and $\mathrm{N} 2$ stage disease ("penetration, N2"), (2) true peritoneal tumor penetration and N1 stage disease ("penetration, N1"), (3) true peritoneal tumor penetration and N0 stage disease ("penetration, N0"), and (4) peritoneal reaction with tumor less than $1 \mathrm{~mm}$ from the peritoneal surface and N0 stage disease (" $<1 \mathrm{~mm}, \mathrm{~N} 0$ ")

( $p=0.017$, log-rank).

Truncation at 42 months, when the patients at risk became less than one-third of the starting group
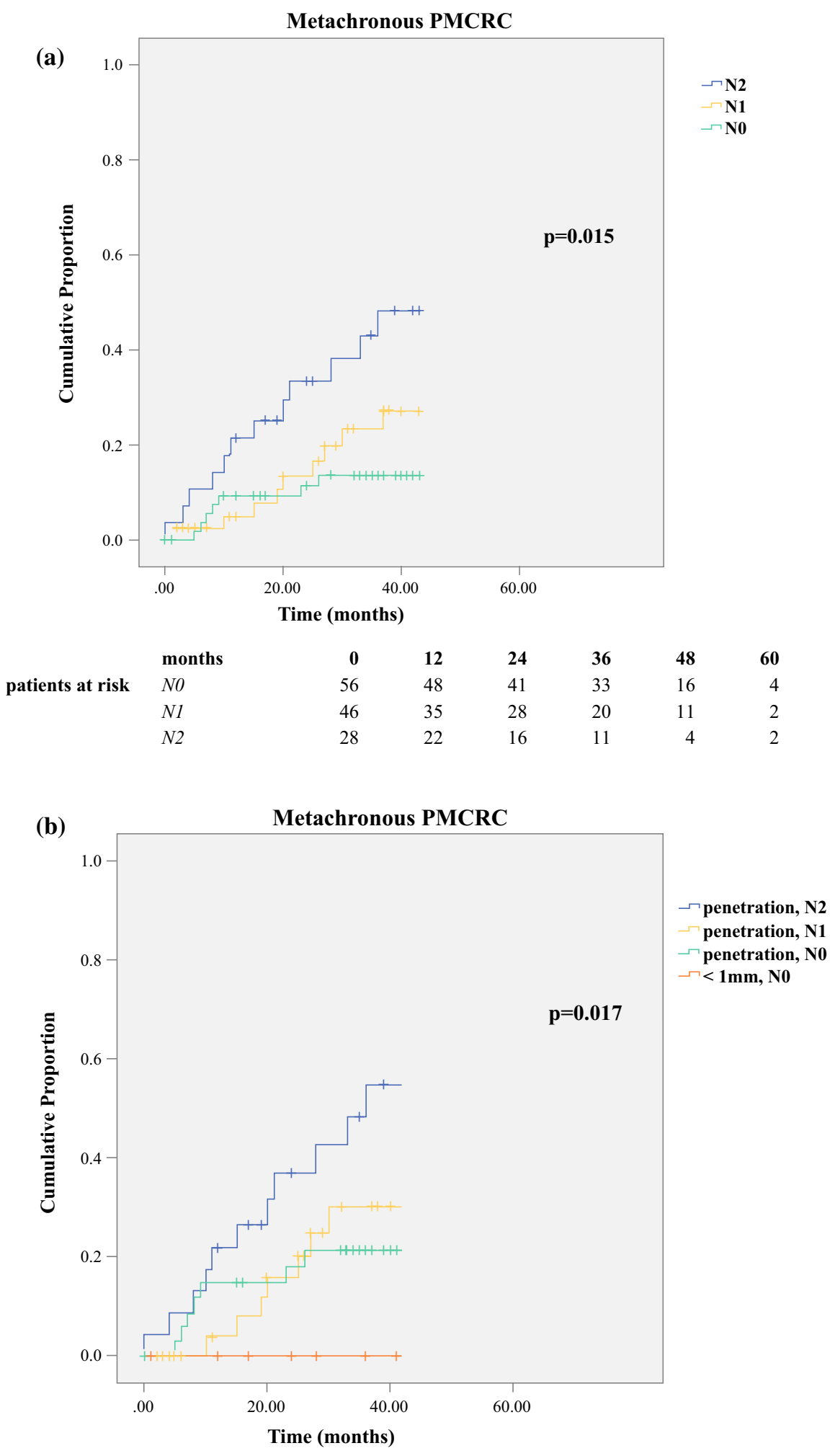

$\begin{array}{llrrrrrr} & \text { months } & \mathbf{0} & \mathbf{1 2} & \mathbf{2 4} & \mathbf{3 6} & \mathbf{4 8} & \mathbf{6 0} \\ \text { patients at risk } & <1 \mathrm{~mm}, \mathrm{NO} & 21 & 20 & 16 & 14 & 7 & 0 \\ & \text { penetration, NO } & 35 & 28 & 25 & 19 & 9 & 4 \\ & \text { penetration, } N 1 & 33 & 24 & 20 & 12 & 7 & 2 \\ & \text { penetration, } N 2 & 23 & 18 & 12 & 8 & 4 & 2\end{array}$


ACKNOWLEDGEMENT The authors acknowledge Steffen Fieuws for improving the methodology and statistical analyses in this study.

CONFLICT OF INTEREST There are no conflicts of interest.

OPEN ACCESS This article is distributed under the terms of the Creative Commons Attribution 4.0 International License (http:// creativecommons.org/licenses/by/4.0/), which permits unrestricted use, distribution, and reproduction in any medium, provided you give appropriate credit to the original author(s) and the source, provide a link to the Creative Commons license, and indicate if changes were made.

\section{REFERENCES}

1. Yang SH, Lin JK, Lai CR, et al. Risk factors for peritoneal dissemination of colorectal cancer. J Surg Oncol. 2004;87:167-73.

2. Jayne DG, Fook S, Loi C, Seow-Choen F. Peritoneal carcinomatosis from colorectal cancer. Br J Surg. 2002;89:1545-50.

3. van Gestel YRBM, Thomassen I, Lemmens VEPP, et al. Metachronous peritoneal carcinomatosis after curative treatment of colorectal cancer. Eur J Surg Oncol. 2014;40:963-9.

4. Segelman J, Granath F, Holm T, et al. Incidence, prevalence, and risk factors for peritoneal carcinomatosis from colorectal cancer. Br J Surg. 2012;99:699-705.

5. Lemmens VE, Klaver YL, Verwaal VJ, et al. Predictors and survival of synchronous peritoneal carcinomatosis of colorectal origin: a population-based study. Int J Cancer. 2011;128(11): 2717-25.

6. de Cuba EM, Kwakman R, van EM, et al. Understanding molecular mechanisms in peritoneal dissemination of colorectal cancer: future possibilities for personalised treatment by use of biomarkers. Virchows Arch. 2012;461:231-43.

7. Sugarbaker PH. Update on the prevention of local recurrence and peritoneal metastases in patients with colorectal cancer. World $J$ Gastroenterol. 2014;20:9286-91.

8. Greene F, Page D, Fleming I. AJCC Cancer Staging Manual. Springer, New York, 2002.

9. Compton CC. Key issues in reporting common cancer specimens: problems in pathologic staging of colon cancer. Arch Pathol Lab Med. 2006;130:318-24.
10. Shepherd NA, Baxter KJ, Love SB. The prognostic importance of peritoneal involvement in colonic cancer: a prospective evaluation. Gastroenterology. 1997;112:1096-102.

11. Ludeman L, Shepherd NA. Serosal involvement in gastrointestinal cancer: its assessment and significance. Histopathology. 2005;47:123-31.

12. Klaver CEL, Musters GD, Bemelman WA, et al. Adjuvant hyperthermic intraperitoneal chemotherapy (HIPEC) in patients with colon cancer at high risk of peritoneal carcinomatosis; the COLOPEC randomized multicentre trial. BMC Cancer. 2015; 15:428.

13. Hompes D, Tiek J, Wolthuis A, et al. HIPEC in T4a colon cancer: a defendable treatment to improve oncologic outcome? Ann Oncol. 2012;23:3123-9.

14. van Santvoort HC, Braam HJ, Spekreijse KR, et al. Peritoneal carcinomatosis in 44 colorectal cancer: occurrence and risk factors. Ann Surg Oncol. 2014;21:1686-91.

15. Honore C, Goere D, Souadka A, Dumont F, Elias D. Definition of patients presenting a high risk of developing peritoneal carcinomatosis after curative surgery for colorectal cancer: a systematic review. Ann Surg Oncol. 2013;20:183-92.

16. Panarelli NC, Schreiner AM, Brandt SM, Shepherd NA, Yantiss RK. Histologic features and cytologic techniques that aid pathologic stage assessment of colonic adenocarcinoma. Am J Surg Pathol. 2013;37:1252-8.

17. Snaebjornsson P, VMHC, LJ, et al. PT4 stage II and III colon cancers carry the worst prognosis in a nationwide survival analysis: Shepherd's local peritoneal involvement revisited. Int $J$ Cancer. 2014;135:467-78.

18. Noura S, Ohue M, Seki Y, et al. Long-term prognostic value of conventional peritoneal lavage cytology in patients undergoing curative colorectal cancer resection. Dis Colon Rectum. 2009;52:1312-20.

19. de Bree E, Koops W, Kröger R, et al. Peritoneal carcinomatosis from colorectal or appendiceal origin: correlation of preoperative CT with intraoperative findings and evaluation of interobserver agreement. J Surg Oncol. 2004;86:64-73.

20. Marin D, Catalano C, Baski M, et al. 64-Section multi-detector row $\mathrm{CT}$ in the preoperative diagnosis of peritoneal carcinomatosis: correlation with histopathological findings. Abdom Imaging. 2010;35:694-700. 